\title{
Complications of CSF shunting in hydrocephalus
}

\author{
Edited by C. Di Rocco, M. Turgut, G. Jallo and J.F. Martinez-Lage, Springer International \\ Publishing, Switzerland: 2015, pp.322, ISBN 978-3-319-09960-6
}

\author{
Mario Giordano ${ }^{1}$
}

Received: 9 March 2015 / Accepted: 18 March 2015 /Published online: 23 April 2015

(C) Springer-Verlag Berlin Heidelberg 2015

In an era of transition between "traditional" CSF shunting and endoscopy, there are still many controversies regarding the treatment of hydrocephalus. In this scenario, the book edited by C. Di Rocco, M. Turgut, G. Jallo, and J.F. Martinez-Lage throws light on the complex topic of CSF shunting complications. Hydrocephalus still remains a disease accounting for a large proportion of the daily neurosurgical practice in children as well as in adults. Due to the multiple etiologies of the disease and its complex impact on the affected subjects, it is not surprising that the high number and different types of complications still weight its surgical treatment.

This multi-authored book is a comprehensive review of the complications that have to be faced in the surgical management of the condition. The authors, who are leaders in the field, have contributed with very good reviews of specific topics. The book is well organized with its practical subdivision in two main sections: complications of extrathecal and intrathecal shunts including discussions about prevention, pathophysiology, diagnostic approaches, and medical and surgical treatment.
Usually, a limitation of medical books is the delay between submission and publication that makes it impossible for authors to provide information on the most recent scientific researches. Fortunately, in this case, the authors include in the volume particularly updated material that involve newly published studies.

The book's 24 chapters are generally well referenced, and the remarkable advances in treatment of hydrocephalus that have been achieved over the past few years are well covered. This volume should be of interest to a large readership including neurosurgeons, residents, neurologists, and pediatricians and could help to increase awareness of the specific features of CSF shunting.

Indeed, in a very few fields of neurosurgery, can a correct management of a disease reach a so high percentage of cure than the surgical treatment of hydrocephalus. In the same time, however, rarely preventable complications and their late recognition or inappropriate treatment may jeopardize the quality of life of the affected subjects. As usual for Springer publications, the quality of printing and the rich iconography with a large number of illustrations both in $\mathrm{B} / \mathrm{W}$ and color add a further charm to the book.
Mario Giordano

mario.giordano@alice.it

1 Department of Neurosurgery, International Neuroscience Institute, Rudolf Pichlmayr Str. 4, Hannover 30625, Germany 